\title{
Diagnostic problems and immune contexture in a case of cecal medullary carcinoma
}

Sofia-Eleni Tzorakoleftheraki ${ }^{1}$, Triantafyllia Koletsa ${ }^{1, *}$, Christos Poulios ${ }^{1}$, Christoforos S. Kosmidis ${ }^{2}$, Aikaterini Foka-Karagiannopoulou ${ }^{1}$, Valentini Tzioufa ${ }^{1}$ and Ioannis Kostopoulos ${ }^{1}$

${ }^{1}$ Pathology Department, Faculty of Medicine, Aristotle University of Thessaloniki, Greece

2 Surgical Clinic, Faculty of Medicine, Aristotle University of Thessaloniki, AHEPA Hospital, Thessaloniki, Greece

\begin{abstract}
Medullary carcinoma (MC) of the colon has recently been described as a separate variant of colorectal carcinoma, associated with microsatellite instability. Despite its morphological similarities to poorly differentiated adenocarcinomas (PDAs) and neuroendocrine carcinomas (NECs), the prognosis seems to be more favorable. A case of an adult female patient who presented with obstructive ileus due to a right-sided colon mass is reported. A specimen of right hemicolectomy was received and a large tumor was observed at the cecum. Tumor sections revealed a poorly differentiated carcinoma, without elements of intestinal differentiation on histologic or immunohistochemical examination. Further investigation lead to the diagnosis of MC of the cecum. The majority of lymphocytic populations of the microenvironment were granzyme B and T-cell intracellular antigen-1 (TIA-1) positive, with an abundant intraepithelial compartment. There were only a few Forkhead box P3(FOXP3) positive T-regulatory cells. It is emphasized that an undifferentiated large bowel carcinoma characterized by black-tan color on gross examination, expressing epithelial keratins and calretinin, proven to be microsatellite unstable with abundant intratumoral cytotoxic lymphocytes should be diagnosed as MC.
\end{abstract}

Keywords: colorectal cancer; microsatellite instability; T-regulatory cells; T-cytotoxic cells; calretinin

\section{Introduction}

As of 2010, medullary carcinoma (MC) has entered the WHO classification of tumors of the digestive system as a distinct subtype of colorectal carcinoma. It is characterized by a solid pattern of neoplastic cells with eosinophilic cytoplasm and nuclei with coarse distribution of chromatin. Intraepithelial lymphocytes are a distinct feature of this subtype [1]. Some authors suggest that diagnosis requires the presence of microsatellite instability, although there is a percentage of tumors that do not show mismatch repair deficiency [2].

Studies based on registry data reveal that MCs account for $0.05-0.08 \%$ of all colorectal carcinomas. On the other hand, studies including pathologically reviewed cases show that the incidence is considerably higher, up to $4.3 \%$. More reports state that selection bias in association with the recent recognition of the entity, lead to its low rate [2]. MCs exhibit an increasing incidence with age and are more common in older patients with a mean age of 71 years $[3,4]$. The majority of individuals are women and the proximal colon is the most frequent localization, with a higher percentage of tumors found at the cecum [3].

MCs exhibit better prognosis in opposition to neuroendocrine carcinomas (NECS) and poorly differentiated adenocarcinomas (PDAs), a fact that shows the importance in distinguishing these entities [3, 4]. The tumor-immunoregulatorymicroenvironment of this unique entity may contribute to its more favorable outcome [5]. Herein, the diagnostic challenges and the lymphocytic composition of a case of MC are presented.

\section{Materials and methods}

A 79-year-old woman presented at the hospital with obstructive ileus. The patient reported fatigue and weight loss of $10 \mathrm{~kg}$ within 7 months. There was no personal or family history of colon polyposis or cancer. Routine laboratory blood tests showed anemia. A Computer Tomography (CT) scan was performed and demonstrated

*Corresponding author: Triantafyllia Koletsa, Pathology Department, Faculty of Medicine, Aristotle University of Thessaloniki, University Campus, 54124, Thessaloniki, Greece, Tel: +302310999228; Fax: +302310999229; Email: koletsa@med.auth.gr

Received 16 April 2017 Revised 05 June 2017 Accepted 15 June 2017 Published 22 June 2017

Citation: Tzorakoleftheraki SE, Koletsa T, Poulios C, Kosmidis CS, FokaKaragiannopoulou A, Tzioufa V, Kostopoulos I. Diagnostic problems and immune contexture in a case of cecal medullary carcinoma. J Cancer Res Ther. 2017; 5(6):40-44. DOI: 10.14312/2052-4994.2017-8

Copyright: (c) 2017 Tzorakoleftheraki SE, et al. Published by NobleResearch Publishers. This is an open-access article distributed under the terms of the Creative Commons Attribution License, which permits unrestricted use, distribution and reproduction in any medium, provided the original author and source are credited. 
a large tumor at the cecum without any pathological observations of nearby lymph nodes or peritoneal free fluid. Due to the emergency status of the ileus the patient was immediately scheduled for an operation of right hemicolectomy.

A specimen of right hemicolectomy was received at the pathology department consisting of ascending colon and cecum, totally measuring $22 \mathrm{~cm}$ in length and $9 \mathrm{~cm}$ in greatest diameter and terminal ileum, measuring $7.2 \mathrm{~cm}$ in length and $2 \mathrm{~cm}$ in greatest diameter. A protruding, ulcerated, black-tan colored tumor was observed at the cecum. The tumor measured $8.5 \mathrm{~cm}$ in greatest diameter and demonstrated an expansive growth pattern. Twenty five lymph nodes were retrieved from the pericolic fat tissue. Tissue sections were fixed in 10\% formalin buffer and embedded in paraffin blocks. Unstained sections were taken for histochemical and immunohistochemical analysis.

\section{Results}

Hematoxylin and eosin stained sections from the tumor showed an ulcerated malignant neoplasm consisting of uniform, round to polygonal cells, with eosinophilic cytoplasm and high nuclear to cytoplasmic ratio (Figure 1a). The nuclei were vesicular with small nucleoli. Neoplastic cells were organized in nests and trabecular formations (Figure 1a). There was no evidence of glandular differentiation. Foci of tumor necrosis and abundant mitoses were found. The tumor invaded through the muscularis propria into the pericolic fat tissue.

Immunohistochemical analysis was performed in order to classify the tumor and to identify the MSI status. All pertinent data are presented in Table 1. Neoplastic cells were immunoreactive to cytokeratin (CK) 8/18, epithelial membrane antigen (EMA) and carcinoembryonic antigen (CEA). However, markers considered evidential of intestinal differentiation, such as CK20, caudal type homeobox 2 (CDX2), mucin (MUC) 1 and MUC2 were negative. The adjacent normal colonic epithelium served as positive internal control for the aforementioned stains. Diffuse cytoplasmic and nuclear positivity of neoplastic cells for calretinin was observed (Figure $1 \mathrm{~b}$ ). Stains for CK5/6 and CK7 did not reveal positivity. There was no evident neuroendocrine differentiation, verified by the absence of staining for neuroendocrine markers (chromogranin, synaptophysin, cluster of differentiation (CD) [56]). Additional stains, such as CD10, CD30, latent membrane protein (LMP) 1, hepatocyte paraffin (HepPar) 1, alpha fetoprotein (aFP), cancer antigen (CA) 125, Estrogen receptor (ER), progesterone receptor (PR), S-100 protein, human melanoma black (HMB) 45 antigen and Melan-A antigen were negative. Mismatch repair protein deficiency was evaluated by performing the corresponding immunohistochemical panel (MutL homolog 1 (MLH1), postmeiotic segregation increased 2 (PMS2), mutS homolog (MSH) 2, MSH6), where lymphocytes and colonic mucosa were useful as internal control. Of the four markers, MLH1 (Figure 1c) and PMS2 exhibited loss of nuclear expression; hence the tumor presented high levels of microsatellite instability.

Variable peritumoral lymphocytic infiltration was present, as highlighted by CD45 antigen, proven to be predominantly T8. There were numerous intratumoral and peritumoral TIA-1 and granzyme B positive cells (Figure 1d, e), comprising the majority of tumor lymphocytes. Perforin was less expressed. Immunostains for CD25 and FOXP3 revealed only few T-regulatory cells (Figure 1f). CD56 positive cells were observed in close relation to neoplastic cells, although they accounted for $<1 \%$ of the lymphocytic population.
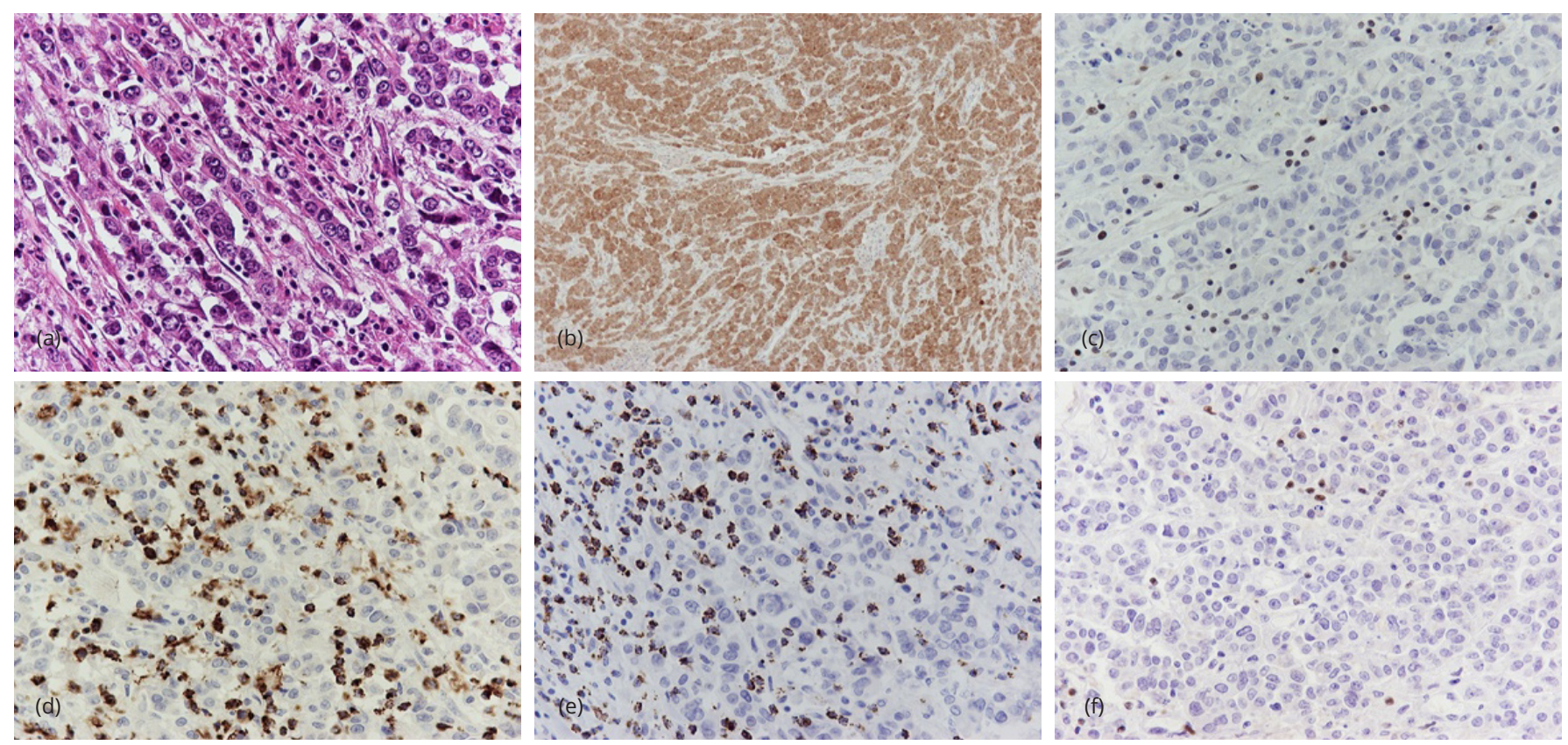

Figure 1 Trabeculae of undifferentiated neoplastic cells (a) positive to calretinin (b) and negative to MLH1 (c) antibodies. TIA-1+ (d) and Granzyme B+ (e) cytotoxic intratumoral cells outnumbered FOXP3+ (f) T-regulatory cells. (a: HE X400; b: IHC X100; c-f: IHC X400). 
Table 1 List of all antibodies used along with the antibody's clone, source, titer and pretreatment.

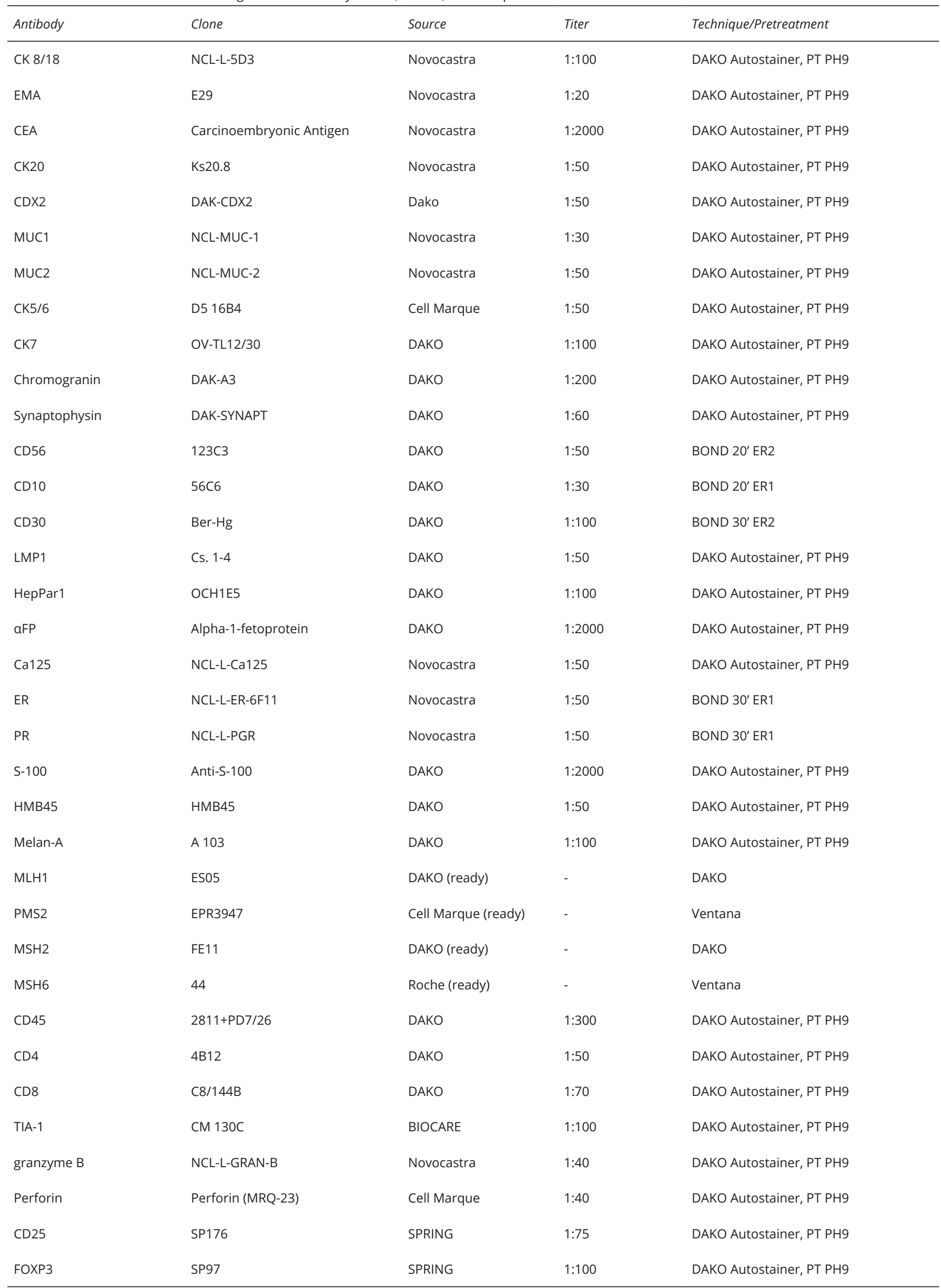


All twenty five of lymph nodes were free of metastases. Taken together, those findings suggested medullary carcinoma of the cecum, stage B2 according to AstlerColler system and T3NOMO according to TNM classification. The patient didn't receive any additional therapy and she remains free of recurrence or metastasis after two years of follow up.

\section{Discussion}

MC of the colon, also described as large cell adenocarcinoma with minimal differentiation or solid-type poorly differentiated carcinoma, is a specific subtype of carcinoma which illustrates different clinicopathological and molecular features from conventional adenocarcinomas [6]. Due to the morphological overlap of MCs and PDAs or NECs, the distinction between them is quite challenging for the pathologist [7]. In this case, the combination of macroscopic, microscopic and immunohistochemical findings were critical to reach the correct diagnosis. A poorly differentiated tumor may present black color on gross examination. The black color is attributed to the combination of ferrous $\left(\mathrm{Fe}^{2+}\right)$ with the hydrogen sulfide produced by intestinal bacteria [8]. Hence, pathologists should be suspicious of medullary carcinoma on gross examination, if a right-sided colon tumor has black color and expansional growth pattern, without evidence of metastatic disease.

At a microscopic level, MCs consist of nests, sheets and trabeculae of small to large-sized, round to polygonal cells with eosinophilic or amphophilic cytoplasm and rounded, vesicular nuclei with small, prominent nucleoli. Glandular differentiation is known to be absent or minimal $[9,10]$. Mitotic figures, apoptotic bodies and necroses might be present [3]. Variable peritumoral or intratumoral lymphocytic infiltration is noted, a feature considered of basic importance in order to diagnose this subtype [11].

Inaddition, criticalexamination oftheimmunohistochemical results is required. Both MCs and PDAs may show evidence of neuroendocrine differentiation. In terms of differentiating those carcinomas from NECs, the latter show diffuse immunopositivity for the majority of neoplastic cells when stained with synaptophysin and chromogranin, whereas MCs and PDAs might exhibit only focal positivity to a minority of the population. Endocrine differentiation does not affect the prognosis and is probably related to the grade of the tumor [3]. In the presented case no neuroendocrine differentiation was observed with the aforementioned stains.

Likewise, intestinal differentiation was absent, as shown by using immunostains for CK20, CDX2, MUC1 and MUC2. Globally, in contrast to CDX2, other intestinal differentiation markers, such as MUC1 and TFF3 are expressed adequately, suggesting the sustainment of intestinal differentiation at some level [12]. MUC4 shows comparable results with TFF3; hence it is regularly expressed in MCs [2]. MUC5A and MUC2 are not expressed as often as MUC1 [7]. As observed in this case, strong positivity for EMA and calretinin is frequently found in MCs [12]. CEA exhibits variable cytoplasmic or membranous positivity [9]. MCs present high levels of microsatellite instability. Even though the MSI-H status is not sensitive, it is a highly specific parameter for this entity [13]. It is a predictive marker, given the fact that it can predict the response to chemotherapy with 5-fluorouracil and irinotecan. It is also reported as a prognostic marker, because of its relation to better survival [7]. MSI-H carcinomas exhibit a better survival rate than stable tumors regardless of the stage or the clinical and pathologic findings [14]. MCs exhibit more favorable prognosis compared with other MSI-H tumors [2]. However, the possibility of metachronous large bowel carcinoma is increased and patient follow up is required [14]. The better prognosis of MSI tumors has also been attributed to intraepithelial T-cells presence in response to the expression of neo-antigens on the cell surface [15]. It has been well established that high densities of cytotoxic T-cells were associated with better prognosis [16]. Besides granzyme B, TIA-1 seems to be a good marker for estimating cytotoxic cells. Furthermore, the intraepithelial T-regulatory cell population has been reported to increase in MCs [5] compared to other subtypes of colorectal carcinomas. In our case there wasn't any remarkable increase of FOXP3 positive cells.

The prognostic impact of increased number of FOXP3 positive intraepithelial lymphocytes in colorectal carcinomas is controversial $[16,17]$, but not in medullary ones [5]. Taking into account the excellent prognosis in our case it can be hypothesized that it is not the high FOXP3 positive cell count that contributes to better prognosis of MCs, but the high ratio of T- cytotoxic/T- regulatory cells. However, this could not be concluded from a single case and it could be of value to compare MC cases with different FOXP3 positive cell counts. In a recent published analogous to this case report [18] the clinical and histological findings were equivalent to those presented herein, having similar free of disease period ( 24 and 28 months, respectively) despite there being no post-surgical treatment in our case. However, the T-cell subgroups of the microenvironment were not mentioned in that case.

\section{Conclusions}

In spite of the histologic appearance and the advanced stage when diagnosed, MCs exhibit more favorable prognosis compared with PDAs and NECs. The distinction between these tumors is challenging but crucial in order to evaluate the prognostic and therapeutic parameters accurately. In this report the macroscopic and microscopic findings of this tumor are discussed. Tumor cells did not exhibit intestinal differentiation immunohistochemically. However, a black-tan tumor on gross examination, expressing epithelial keratins and calretinin, proven to be microsatelliteunstablewithabundantintratumoralcytotoxic lymphocytes, is nothing else but colorectal medullary carcinoma. Intratumoral T- cytotoxic cells, positive to CD8, TIA-1 and granzyme B markers are consistently abundant, whereas FOXP3 positive T-regulatory cells may vary among different cases of this entity, presenting however a high ratio of CD8+/FOXP3+ cells; it is noted that the later finding may affect the prognosis or treatment options, taking into consideration the excellent outcome in this case, but further investigation in a large series is needed. 


\section{Conflict of interest}

The authors declare that there is no conflict of interest regarding the publication of this paper.

\section{References}

[1] Hamilton SR, Bosman FT, Boffeta P, llyas M, Morreau H, et al. Carcinoma of the colon and rectum. In: Bosman FT, Carneiro F, Hruban RH, Theise ND, editors. WHO classification of tumours of the digestive system. 4th ed. Lyon: IARC Press. 2010.

[2] Knox RD, Luey N, Sioson L, Kedziora A, Clarkson A, et al. Medullary colorectal carcinoma revisited: A clinical and pathological study of 102 cases. Ann Surg Oncol. 2015; 22(9):2988-2996.

[3] Wick MR, Vitsky JL, Ritter JH, Swanson PE, Mills SE. Sporadic medullary carcinoma of the colon. Am J ClinPathol. 2005; 123(1):56-65.

[4] Thirunavukarasu P, SathaiahM, SinglaS, Sukumar S, Karunamurthy A, et al. Medullary carcinoma of the large intestine: A population based analysis. Int J Oncol. 2010; 37(4):901.

[5] Friedman K, Brodsky AS, Lu S, Wood S, Gill AJ, et al. Medullary carcinoma of the colon: A distinct morphology reveals a distinctive immunoregulatory microenvironment. Mod Pathol. 2016; 29(5):528541.

[6] Pyo JS, Sohn JH, Kang G. Medullary carcinoma in the colorectum: A systematic review and meta-analysis. Hum Pathol. 2016; 53:91-96.

[7] Fiehn AMK, Grauslund M, Glenthøj A, Melchior LC, Vainer B, et al. Medullary carcinoma of the colon: Can the undifferentiated be differentiated? Virchows Arch. 2015; 466(1):13-20.

[8] Wang L, Warner NE, Sherrod AE. Pathologic quiz case: A 79-year-old woman with a black, ulcerated cecal tumor and 3 negative guaiac test results. Medullary adenocarcinoma of the colon, poorly differentiated. Arch Pathol Lab Med. 2005; 129(1):113-114.

[9] Jessurun J, Romero-Guadarrama M, Manivel JC. Medullary adenocarcinoma of the colon: Clinicopathologic study of 11 cases. Hum Pathol. 1999; 30(7):843-848.

[10] Rüschoff J, Dietmaier W, Lüttges J, Seitz G, Bocker T, et al. Poorly differentiated colonic adenocarcinoma, medullary type: clinical, phenotypic, and molecular characteristics. Am J Pathol. 1997; 150(5):1815-1825.

[11] Chetty R. Gastrointestinal cancers accompanied by a dense lymphoid component: an overview with special reference to gastric and colonic medullary and lymphoepithelioma-like carcinomas. J Clin Pathol. 2012; 65(12):1062-1065.

[12] Winn B, Tavares R, Fanion J, Noble L, Gao J, et al. Differentiating the undifferentiated: immunohistochemical profile of medullary carcinoma of the colon with an emphasis on intestinal differentiation. Hum Pathol. 2009; 40(3):398-404.

[13] Alexander J, Watanabe T, Wu TT, Rashid A, Li S, et al. Histopathological identification of colon cancer with microsatellite instability. Am J Pathol. 2001; 158(2):527-535.

[14] Lanza G, Gafà R, Maestri I, Santini A, Matteuzzi M, et al. Immunohistochemical pattern of MLH1/MSH2 expression is related to clinical and pathological features in colorectal adenocarcinomas with microsatellite instability. Mod Pathol. 2002; 15(7):741-749.

[15] Tougeron D, Fauquembergue E, Rouquette A, Le Pessot F, Sesboüé $\mathrm{R}$, et al. Tumor-infiltrating lymphocytes in colorectal cancers with microsatellite instability are correlated with the number and spectrum of frameshift mutations. Mod Pathol. 2009; 22(9):1186-1195.

[16] Galon J, Pagès F, Marincola FM, Angell HK, Thurin M, et al. Cancer classification using the immunoscore: A worldwide task force. J Transl Med. 2012; 10:205.

[17] Shang B, Liu Y, Jiang SJ, Liu Y. Prognostic value of tumor-infiltrating FoxP3+ regulatory $T$ cells in cancers: A systematic review and metaanalysis. Sci Rep. 2015; 5:15179.

[18] Martinotti M, Cirillo F, Ungari M, Tanzi G, Rolando G, et al. Microsatellite instability in medullary carcinoma of the colon. Rare Tumors. 2017; 9(1):6541. 